\title{
Novel design for a phase lla placebo-controlled, double-blind randomized withdrawal study to evaluate the safety and efficacy of CNV1014802 in patients with trigeminal neuralgia
}

Joanna M Zakrzewska ${ }^{*}$, Joanne Palmer ${ }^{2}$, Dominik A Ettlin², Mark Obermann ${ }^{4}$, Gerard MP Giblin², Valerie Morisset ${ }^{2}$, Simon Tate ${ }^{2}$ and Kevin Gunn ${ }^{2}$

\begin{abstract}
Background: Trigeminal neuralgia (TN) is a rare severe unilateral facial pain condition. Current guidelines in trigeminal neuralgia management recommend sodium channel blockers - carbamazepine or oxcarbazepine - as the first-line treatment. However, the currently available drugs are often associated with poor tolerability resulting in sub-optimal pain control. CNV1014802 is a novel sodium channel blocker that is being assessed in the treatment of trigeminal neuralgia. Due to the severity of the condition, it is not ethical to conduct a traditional placebo-controlled randomized controlled trial. It is also difficult to use an active control such as carbamazepine, the current gold standard, because of its complex pharmacology and potential for drug interactions.

Methods/Design: The trial uses a randomized withdrawal design to assess efficacy in this rare condition. There is a 21-day open-label phase followed by a randomized 28-day placebo-controlled phase for responders. Thirty patients will be randomized. The primary outcome measure will be pain relief, but secondary measures of quality of life will be of significant importance given the effect of this condition on activities of daily living. Safety and adverse event endpoints are described.

Discussion: There have been very few well-controlled, randomized, placebo-controlled studies in trigeminal neuralgia, and the majority of drugs have had other primary uses. Due to the severity of the pain, minimizing the time a patient is administered placebo was a key factor in designing this study. This study will not only provide data on the efficacy of CNV1014802 in trigeminal neuralgia, but will also provide information on the effectiveness and acceptability of a novel trial design in trigeminal neuralgia.
\end{abstract}

Trial registration: Trial number NCT01540630.

Keywords: Trigeminal neuralgia, Double-blind randomized withdrawal study, Sodium channel blocker, Neuropathic pain

\footnotetext{
* Correspondence: j.zakrzewska@ucl.ac.uk

${ }^{1}$ Facial pain unit, Division of Diagnostic, Surgical and Medical Sciences, Eastman Dental Hospital, UCLH NHS Foundation Trust/University College London, 256 Gray's Inn Road, WC1X 8LD, London, UK

Full list of author information is available at the end of the article
} 


\section{Background}

Trigeminal neuralgia (TN) is an uncommon episodic severe facial pain condition with an incidence of 4.0 to 4.7 per 100,000 persons per year [1,2]. Trigeminal neuralgia can appear at any age, but disease onset is over 40 years of age in over $90 \%$ of cases with peak onset between 50 and 70 years of age [2,3]. However, recent data from primary care practice data validated by experts suggest an incidence rate of 12.6 per 100,000 person years with a mean age at diagnosis of 51.5 (SD 17.6) with a female predominance $71 \%$ [4]. The classification system of the International Headache Association aims at establishing TN diagnostic criteria based on etiology [5]. Yet, there is a problem in distinguishing primary and secondary $\mathrm{TN}$, because unless a patient with normal neuroimaging comes to an operation, it remains unclear if his/her TN is caused by a vascular compression. To adjust for this diagnostic uncertainty, the term "classical" neuralgia is currently used for cases with normal neuroimaging and potential compression of the proximal trigeminal nerve root by a vascular loop. Classical TN is the most common TN type, and it is thought that secondary demyelination, probably mediated by microvascular ischemic damage, results in a lowered excitability threshold of affected neurons. This promotes inappropriate ectopic generation of spontaneous nerve impulses together with abnormal nonsynaptic ephatic transmission to adjacent neurons [6,7].

Unlike many other neuropathic pains, $\mathrm{TN}$ results in recurrent paroxysms of short-lasting but very severe pain in the distribution of one or more branches of the trigeminal nerve. In patients with the classical type, the attacks come on suddenly, last up to $2 \mathrm{~min}$ and disappear suddenly. Between attacks, patients are usually asymptomatic, although there may be a slight after pain [8]. Numerous attacks occur a day, but currently there are no studies detailing how many paroxysms occur a day. In a recent RCT of botulinum toxin A in $42 \mathrm{TN}$ patients, the frequency of paroxysms was measured, and the average was 20 a day, but the range was 4-100 [9]. Patients also report that these can be so frequent that it seems like one long attack. The attacks can vary in severity and be described as shooting, electric shocks scoring maximum scores on scales or just "twinges." These attacks can then go on for weeks or months. Especially in the early stages of the disorder, it is very common for the attacks to stop completely and for patients to have weeks, months or even years of no pain [10]. However, over time the remission periods get shorter. Currently, there are no natural history studies or known prognostic factors to help determine how long these periods can be, although Taylor et al. [11] showed that carbamazepine became less effective with time. This may be due to the natural course of the disease, but may also be vested in the autoinduction pharmacological properties of carbamazepine itself. Pain is provoked by light touch activities, e.g., washing, eating, talking and cold winds, but spontaneous attacks of pain also occur. One study that measured the occurrence of evoked or spontaneous pain suggested that oxcarbazepine was more effective at reducing the former [12]. Although attacks of pain occur at night, these are less common. Thus, even when pain free, patients live in fear of pain return. These features therefore make it difficult to evaluate the effect of treatments. The diagnostic criteria for TN most frequently used are those of the International Headache Society, the International Classification of Headache Disorders (ICHD) [13], and these have now been updated with clearer specification of the variants of TN, i.e., those patients who may have in addition to the shooting stabbing pain a more prolonged pain, and these are thought to have a potentially different pathophysiology [14].

Current guidelines in trigeminal neuralgia management recommend sodium channel blockers such as carbamazepine or oxcarbazepine as the first-line treatment for pain control. However, these agents are often poorly tolerated [15] and often require lengthy dose escalation, resulting in sub-optimal efficacy, and some result in significant drug interactions and require careful monitoring. Other potential second-line therapies include lamotrigine, pregabalin, gabapentin and baclofen; there is limited evidence to support the use of these agents $[15,16]$. Unlike other medications used in chronic pain, these drugs often result in complete pain relief, not just 50\%, especially in the earlier stages of the disorder.

All the drugs used to date have been initially developed for other uses, principally epilepsy, so dosage scales have had to be adapted. A review of RCTs and Cochrane systematic reviews (SR) shows that the major side effects reported from antiepileptic drugs (AED) were drowsiness from $100-4 \%$, dizziness or vertigo $47-3 \%$, gastrointestinal (GI) 57 to- $8 \%$, mood changes $2 \%$, dry mouth or taste change $4-2 \%$, and headaches $4 \%$. The SR on carbamazepine (CBZ) [17] reported 40-60\% would have side effects. However, no study provides details on how these events were measured or quantified. Studies in healthy volunteers do show that AED drugs, especially the older ones such as CBZ, do result in cognitive impairment, although this is generally modest but can have clinical significance [18]. Memory, especially those tasks with an attentional component, is reduced when on CBZ $[19,20]$. However, it also needs to be noted that pain has an impact on cognitive function [21].

Thus, a well-tolerated sodium channel blocker that can be administered at an effective dose with no titration and good tolerability may address some of the unmet needs of this patient group. 
CNV1014802 is a peripherally and centrally acting agent that inhibits sodium channels in a state-dependent fashion. CNV1014802 shows selectivity for the Nav1.7 subtype over the other subtypes tested (Nav1.1, Nav1.2, Nav1.3, Nav1.5, Nav1.6 and TTX-R), for both the resting and depolarized states. The greater block of Nav1.7 is particularly enhanced at concentrations below $1 \mu \mathrm{M}$, where the free exposure in animal models and human clinical doses lies.

In addition, the amount of block by CNV1014802 increases significantly and in a similar way with the frequency of stimulation for Nav1.7, Nav1.2 and Nav1.6. Combining all aspects of the pharmacology of CNV101802 at sodium channels, the block is more activity-driven at Nav1.2 and Nav1.6 than it is at Nav1.7, where it is substantial even at lower levels of activity of the channel. The block at Nav1.5 and TTX-R is significantly weaker.

Consistent with its mechanism of action, CNV1014802 will preferentially target and inhibit higher frequencies of firing (from $10 \mathrm{~Hz}$ onwards) that are attained following noxious stimuli or occur in chronic pain conditions or during seizure activity.

CNV1014802 has also been shown to selectively and reversibly inhibit the monoamine oxidase (MAO)-B enzyme, with no effect on MAO-A. One hundred percent inhibition of MAO-B was achieved with doses of $75 \mathrm{mg}$ and above in the clinic studies.

A genetic substrate for neuropathic pain is an accepted hypothesis in the scientific community. Recently, sodium channel gene mutations causing cell hyperexcitability have been identified in groups of patients with painful neuropathy $[22,23]$. Calcium channelopathies have also been linked to migraine and epilepsy [24]. Given the importance of sodium and calcium channels in the generation, propagation and plasticity of pain signals, it is proposed to genotype five sodium channel (Nav1.1, Nav1.2, Nav1.3, Nav1.6 and Nav1.7) and two calcium channel (Cav2.2 and Cav2.1) genes in all patients entering the study to explore whether mutations in these genes are present in $\mathrm{TN}$ and whether these are related to response to treatment with CNV1014802.

CNV1014802 has completed extensive phase I studies with single and repeated doses in 166 healthy volunteers.

Moore et al. [25] in their analysis of analgesic drugs propose that high failure rates of drugs must be expected especially in chronic pain, that a radical re-think is necessary in the design of analgesic trials and that an enhancement enriched randomized withdrawal design may be the way forward, but currently they are poorly understood in part because few have been carried out.

Thus, the aim of this study is to design and test a protocol to evaluate the efficacy of a new sodium channel blocker, CNV1014802, in TN, which takes into account the difficulties encountered in previous trials in this area. An additional aim, if study participants agree, is genotyping for possible sodium and calcium channelopathies. The protocol was designed and approved prior to the SPIRIT 2013 statement, but will try to adhere to the published check list [26].

\section{Methods and design}

A review of the literature was first carried out and showed that clinical trials in $\mathrm{TN}$ are challenging, and many of the published trials in this disorder have recruited low numbers of patients and have used variable designs and outcome measures, resulting in inconclusive outcomes [17,27-29]. Traditional placebo-controlled studies (crossover or parallel group) are difficult to run in this condition, as significant numbers of patients will be exposed to placebo for an extended duration. Using active controls is difficult as the gold standard drug carbamazepine is metabolized through the liver and so takes time to be eliminated and for the liver enzymes to return to normal function, thus necessitating extended washout periods. Because of the severe nature of the pain, patients are unlikely to accept extended periods of no treatment or use of placebos. Two-stage enhancement enriched randomized withdrawal designs (EERW) have recently been proposed as an alternative strategy for determining the effectiveness of analgesic drugs [30,31]. Although bias will occur as only responders are enrolled into the randomized part of the trial and they may also guess whether they are on the active drug or not, it does offer the patients a rapid termination and return to previous medication. Response to the open label part of the study also allows any adjustments to be made to the dosage and to evaluate whether the drug has any potential in the individual patient. A recent study using levetiracetam for TN showed poor efficacy in the open label phase, so the randomized trial was not started [32]. With a disorder that results in unexpected remission periods, it is essential to ensure that at the end of the trial it is established that the disease has not gone into remission.

Outcome measures were chosen according to the recently published recommendations from the International Association for the Study of Pain committee [33], which suggest the following areas should be assessed using psychometrically tested tools: pain intensity, physical and emotional functioning, participants global improvement and satisfaction, symptoms and adverse events, and participants disposition. Trigeminal neuralgia is unusual in that it is considered a chronic pain, but the pain is very clearly episodic. Attacks can vary in number and anecdotally patients have reported that a significant outcome is to have a reduction in pain attacks even if their intensity remains high. In an RCT of use of lamotrigine in $\mathrm{TN}$, a composite score was obtained that included 
the number of bursts of pain, i.e., not individual stabs of pain but paroxysms of pain (none, 1-3, 4-7, 8-12, 13-20, over 20), severity of pain (no pain, mild, moderate, severe) and degree of pain relief (complete, good, moderate, slight, none) [34]. Thus, the number of paroxysms daily would also be used as an outcome measure, although there are no data on the number of attacks expected, but a $50 \%$ reduction would be considered successful. The pain intensity of each paroxysm is assessed on an 11-point numerical rating scale (PI-NRS). The Brief Pain Inventory-Facial (BPI-Facial), which is a validated 18-item rating scale for facial pain in a TN population [35], is also included as a composite assessment of efficacy, which includes the impact of pain on quality of life.

Special emphasis will be placed on adverse events as drugs such as carbamazepine are highly effective, but their tolerability is very poor, thus limiting their use. A scale that has been developed and tested in large numbers of patients with epilepsy is the adverse events profile (AEP) by Baker et al. [36,37]. This would provide more quantifiable data than have previously been reported.

Although measuring cognitive function is complex and it has been suggested that self-administered scales may have a much stronger relationship to participants' mood rather than their objectively measured cognitive performance, some attempt will be made to measure this using the Medical Outcomes Study-Cognitive Scale (MOS-Cog) [38,39].

Genotyping is not mandatory for patients participating in the study.

The trial was designed by the chief investigator (JZ) and the Convergence team with protocol review by two outside neurologists with experience in this disorder and later by two of the authors (MO; DE) Additional file 1 . A brief summary of the trial was presented at a UK Trigeminal Neuralgia support group meeting to determine whether patients would take part in the study and whether the correct outcome measures were chosen. Expert patients were asked to comment on the patient information sheet, consent form and diary card. The study has been approved by regulatory authorities and ethics committees in 11 countries: the UK, Germany, Switzerland, Estonia, France, Italy, Latvia, Lithuania, Romania, South Africa and Spain. The names of the committees are to be found in the Additional file 2, Ethics Committees. Although it is anticipated that patients will be referred in from the community, the study will take place in secondary care centers, which have a special interest in headache and facial pain.

The trial was designed to use an initial dose of $150 \mathrm{mg}$ three times daily with the flexibility to use a higher dose regimen of $350 \mathrm{mg}$ twice daily if appropriate. A review of efficacy data after the first ten patients had completed the open label study was planned to review efficacy and tolerability. If these were acceptable, as judged by a data monitoring committee, then the trial would continue to completion without changing the dose. All investigators and their teams met for 1 day to undertake training and review the protocol, and the operational practicalities of the study including site selection, monitoring and data management were taken over by a contract research organization $(\mathrm{CRO})$.

The outline protocol is available at www.clintrials.gov, trial no. NCT01540630, and gained ethical approval in the UK in September 2011, then subsequently in all the other participating countries. A final harmonized version of the protocol, incorporating all country-specific minor amendments, was available in August 2013.

A double-blind, randomized withdrawal study comparing CNV1014802 with placebo in patients with TN who have successfully responded to CNV1014802 in an initial open-label phase was designed to take into account all the difficulties highlighted in the Methods section. Figure 1 shows the overall design of the study.

\section{Design}

Patients participate in an initial open-label treatment period of 21 days of CNV1014802 using $150 \mathrm{mg}$ three times daily. Responders are then randomized to 28 days of CNV1014802 $150 \mathrm{mg}$ three times daily or placebo.

A responder at the end of the open-label period was defined as a patient with one of the following:

- A $30 \%$ or more decrease in the total number of paroxysms over the last 7 days of the open-label phase as compared to the total number recorded in the 7 -day baseline phase (day -7 to -1 prior to start of study medication)

- A $30 \%$ reduction in the mean severity of pain experienced during the paroxysm over the last 7 days of the open-label phase as compared to the total number recorded in the 7-day baseline phase (day -7 to -1 prior to start of study medication)

- A Patient Global Improvement of Change rating of much improved/very much improved

\section{Randomization and allocation concealment mechanism}

In the initial open-label treatment period, all patients will receive CNV1014802.

Patients will be randomized to CNV1014802 or placebo, in a 1:1 ratio, in accordance with the randomization schedule. The randomization schedule will be generated by the CRO using the PROC PLAN procedure of SAS ${ }^{\circ}$, a validated computer system. Eligible patients will be randomized into the study on day 21 . The randomization schedule will be stratified by whether the patient is on existing pain medication (adjunct) or not (monotherapy). 


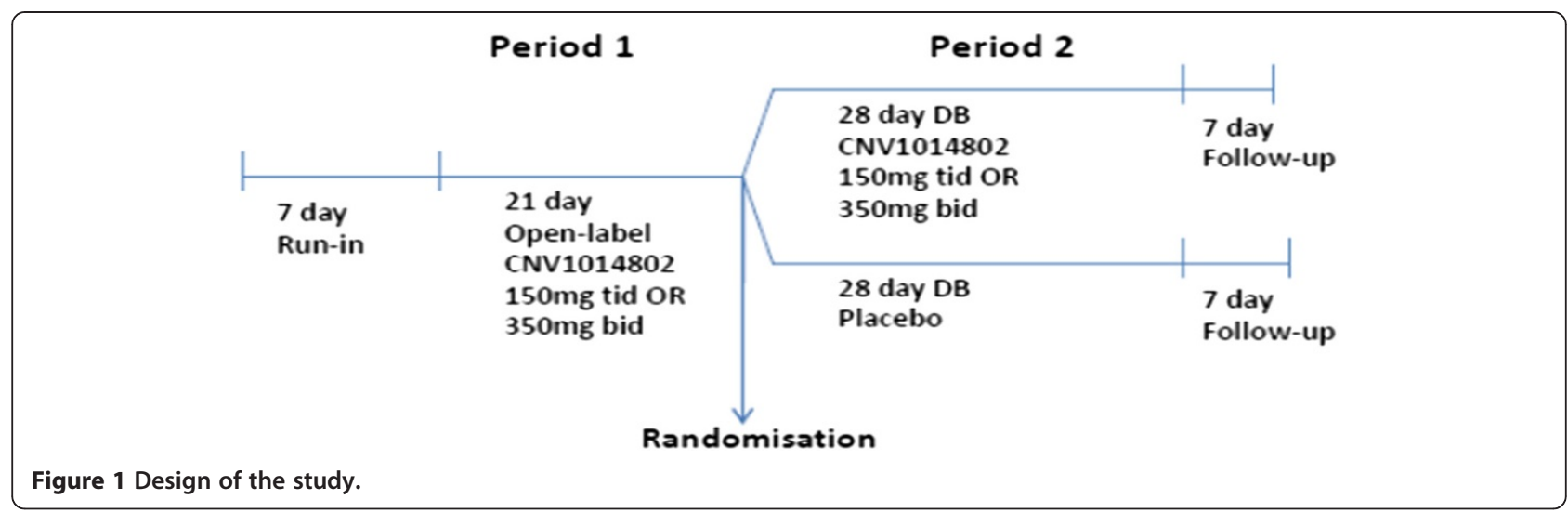

Equal numbers of patient on adjunct and monotherapy are not required.

\section{Outcome measures}

The primary outcome measure of efficacy is the number of treatment failures on CNV1014802 vs. number of treatment failures on placebo throughout the double-blind treatment period. Patients are classified as a treatment failure if they meet one of the following criteria:

i. A 50\% increase in the frequency of paroxysms compared to the final 7 days of the open-label period to more than three paroxysms within a 7-day period

ii. When more than three paroxysms are reported in a 7 -day period, a $50 \%$ increase in the severity of pain experienced in the paroxysms compared to the final 7 days of the open-label period

iii. A Patient Global Improvement of Change rating of much worse/very much worse

iv. The patient discontinues the study because of 'lack of efficacy.'

$\mathrm{v}$. The patient discontinues because of an adverse reaction or poor tolerability considered to be related to the study medication

During the double-blind randomized phase, patients will be evaluated to determine whether they meet the failure criteria at each clinic visit, which will occur every 7 days in the double-blind treatment period. The data for the frequency and severity of the pain will be taken from the daily diaries completed by the patients. Figure 2 is an example of such a diary.

Secondary outcome measures of efficacy are:

- Proportion of failures by week

- Kaplan-Meier analysis of time to failure

- Average change in the pain intensity numerical rating scale PI-NRS by week

- Average change in PI-NRS (best response)

- Median change in the number of paroxysms by week
- Median change in the number of paroxysms (best response)

- Patient and physician global impression of change

- Average change in the Brief Pain Inventory BPI-Facial by week [35]

- Average change in the BPI-Facial (best response)

- Number and severity of paroxysms of pain in the 21-day open-label period, both evoked and spontaneous

- Average 24-h pain intensity numerical rating scale (PI-NRS)

- Patient and Physician Clinical Global Impression of Change

Other measures will include:

- Adverse events, vital signs, ECG and safety laboratory samples will be collected throughout the study

- Adverse event profile: this questionnaire was developed for epilepsy to understand the common AEs associated with epilepsy $[36,40]$, collecting it at screening, day 21 and the last day in the study to try and compare tolerability on CNV1014802 compared to other anti-epileptics they may be taking at screening.

- Medical Outcomes Study-Cognitive Scale (MOS-Cog) will be completed at screening and the end of the study $[38,39]$

- Assessment of blindedness of study medication: Both the patient and clinician will provide a guess as to which medication (CNV1014802 or placebo) was administered during the double-blind treatment period

- Pre- and post-dose plasma concentrations of CNV1014802

- Presence of genetic mutations in sodium channel (Nav1.1, Nav1.2, Nav1.3, Nav1.6 and Nav1.7) or calcium channel (Cav2.2 and Cav2.1) genes

If the patient requires a drug treatment for their $\mathrm{TN}$ pain other than paracetamol, they will be considered a 


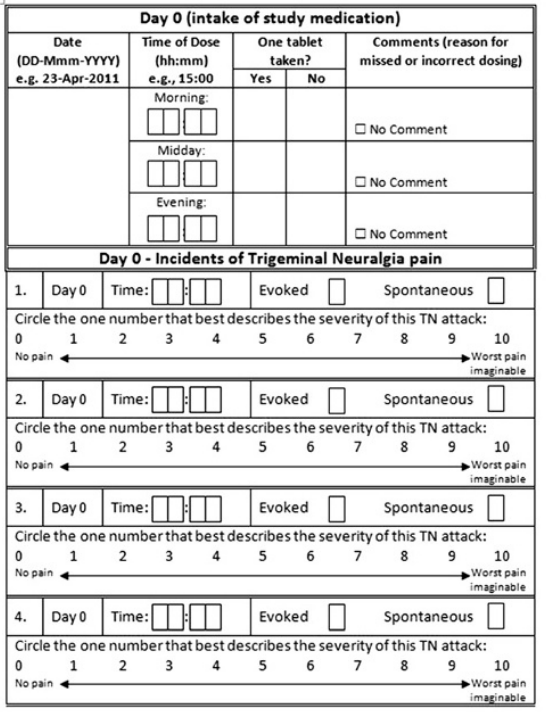

Figure 2 Pain diary completed by the patient on a daily basis.

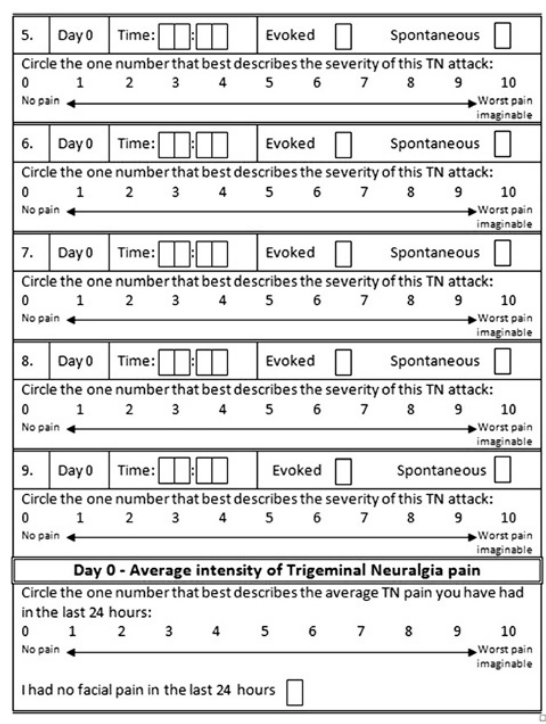

humans has not yet been fully evaluated, a variety of drug types have to be stopped prior to study commencement, and approved concomitant medications must have been stable for at least 3 weeks prior to day 0 . This includes but is not limited to sodium channel blockers or drugs that adversely interact with a monoamine oxidase-B inhibitor: MAOIs, antidepressants, opioids and sympathomimetic agents. Other than gabapentin and pregablin, no other antiepileptic drug (AED) is allowed.

Among the exclusion criteria are patients who are known non-responders to sodium channel blockers at therapeutic doses and those with a history of uncontrolled or poorly controlled hypertension. Patients with a history or presence of significant cardiovascular, gastrointestinal or renal disease or other condition known to interfere with the absorption, distribution, metabolism or excretion of drugs are also excluded.

The rescue medication is paracetamol only to a maximum dose of $4 \mathrm{~g} /$ day.

As the trial is intended to be carried out in multiple countries, it is crucial to ensure that only classical TN patients are recruited and not those whose diagnosis is equivocal and who had tried a large number of drugs with poor response. The data-monitoring committee (DMC) is made up of three clinical investigators with experience in diagnosing patients with TN (JZ, DE, MO), the medical director and study manager from Convergence and from the CRO medical monitor, statistician and project leader. Their remit as well as verifying the diagnosis is to evaluate ongoing efficacy and safety data from the open-label period and to recommend: the dose of CNV1014802 to be used following review of the first ten evaluable subjects completing the open-label metabolized, and that the drug interaction potential in 


\section{Table 1 CNV1014802 in patients with trigeminal neuralgia inclusion and exclusion criteria}

\section{Major inclusion criteria}

A patient will be eligible for inclusion in this study only if all of the following criteria apply:

1. The following diagnostic criteria for trigeminal neuralgia must be met:

Paroxysmal attacks of pain lasting from a fraction of a second to 2 min affecting one or more divisions of the trigeminal nerve

Pain has at least one of the following characteristics:

i. Intense, sharp, superficial or stabbing

ii. Precipitated from trigger areas or by trigger factors

iii. Attacks are stereotyped in the individual patient

There is no clinically evident neurological deficit

Not attributed to another disorder

2. Frequency criteria for numbers of paroxysms:

Patients must have suffered a minimum of 3 or more paroxysms of pain per day, rated at an intensity of 4 or more on the pain NRS, on at least 4 days during the last 7 days prior to entry into the open-label treatment period.

3. Male or female between 18 and 80 years of age inclusive at the time of signing the informed consent.

4. A female patient is eligible to participate if she is of non-childbearing or child-bearing potential and agrees to use one of the contraception methods listed.

5. Male patients must agree to use one of the contraception methods.

6. Body weight $\geq 50 \mathrm{~kg}$ for men and $\geq 45 \mathrm{~kg}$ for women.

7. $\mathrm{BMI} \leq 34.9$.

8. Capable of giving written informed consent, which includes compliance with the requirements and restrictions listed in the consent form. Informed consent must be obtained prior to the commencement of any study-related procedures.

\section{Major exclusion criteria}

A patient will not be eligible for inclusion in this study if any of the following criteria apply:

1. Patients who are known non-responders to sodium channel blockers at therapeutic doses. If patients have previously been unable to tolerate sodium channel blockers and therefore has not been able to take doses within the therapeutic dose range, they may still be included.
3. A positive pre-study hepatitis B surface antigen or positive hepatitis $C$ antibody result within 3 months of screening.

4. History of any liver disease within the last 6 months, with the exception of known Gilbert's disease.

5. History of excessive regular alcohol consumption within 6 months of the study defined as: an average weekly intake of $>28$ units or average daily intake $>4$ units for males; an average weekly intake $>21$ units or average daily intake $>3$ units for females. One unit is equivalent to $8 \mathrm{~g}$ of alcohol: a half-pint $(\sim 240 \mathrm{ml})$ of beer, 1 glass $(125 \mathrm{ml})$ of wine or $1(25 \mathrm{ml})$ measure of spirits.

6. Patients with a history or risk of seizures or a history of epilepsy, head injury or related neurological disorders.

7. Patients with a history of uncontrolled or poorly controlled hypertension

8. History or presence of significant cardiovascular, gastrointestinal, or renal disease or other condition known to interfere with the absorption, distribution, metabolism or excretion of drugs, which, in the opinion of the investigator, may interfere with the study procedures or compromise patient safety.

9. Patients with conditions known to affect cardiac conduction or a personal or familial history of Brugada syndrome.

10. Females of child-bearing potential only: pregnant females as determined by positive urine or serum hCG test at screening or prior to dosing.

\section{Lactating females.}

12. History or presence of any clinically significant abnormality in vital signs/ ECG/laboratory tests, or any medical or psychiatric condition, which, in the opinion of the investigator, may interfere with the study procedures or compromise patient safety.

13. The patient has a history of suicidal ideation and/or suicide attempts.

14. The patient has clinical evidence of recent major depression

(by patient's medical history). period. All patients considered for screening are required to complete a two-page form on diagnostic criteria to be considered by the DMC and approved by at least two of the investigators. The DMC also receives weekly reports on the progress of the trial and numbers required from each center and their progress through the study. It can also terminate the study depending on the efficacy and tolerability of the drug.

All the data from the trial are collected on electronic clinical record forms and anonymized prior to submission, 
and each site is visited by the project lead and local lead from the CRO. All investigators must adhere to Good Clinical Practice (GCP) and have undergone the appropriate training. All the investigational site staff took part in the protocol training program, either face to face or web based.

The study is double-blind and there are strict restrictions on unblinding. The investigator or treating physician may unblind a patient's treatment assignment only in the case of an emergency, when knowledge of the study treatment is essential for the appropriate clinical management or welfare of the patient.

Pharmacovigilance staff acting on behalf of the sponsor may unblind the treatment assignment for any patient with a serious adverse event (SAE). If the SAE requires that an expedited regulatory report be sent to one or more regulatory agencies, a copy of the report, identifying the patient's treatment assignment, may be sent to clinical investigators in accordance with local regulations.

\section{Power calculation}

The aim is to enroll sufficient patients to randomize 30 responders into the double-blind randomized phase of the study. If evaluable data are available from 22 patients entering the randomized phase, assuming a $20 \%$ failure rate for CNV1014802 and a 67\% failure rate for placebo, 11 evaluable patients per treatment arm (22 in total) would provide $80 \%$ power to detect a difference of $47 \%$ between the proportion of failures on CNV1014802 and placebo, assuming a one-sided test with a type I error rate of $5 \%$.

\section{Discussion}

This is the first time that such a design has been used to evaluate a drug for $\mathrm{TN}$. It is also the first time that a drug is being used that has not first been evaluated in epilepsy. Its design conforms with the CONSORT guidelines, which have been shown to improve the quality of trial reporting [41], and although conceived before the statement on defining the standard protocol for clinical trials was published, it does conform to these [26]. Drug trials in TN are rare, heterogeneous and often contain serious methodological issues. Only 11 randomized placebo controlled trials on classical TN have been conducted since 1967. These trials recruited very different patient numbers (ranging from 3 to 77 participants), often embedding TN patients into a broader facial pain patient spectrum study to gain more statistical power. Formal power calculations were generally not provided in most previous TN trials. The study by Gilron et al. [42] is an extreme example of recruitment difficulties that all TN trials face. This placebo-controlled, multiple cross-over pilot study included only three patients. The present study is also small and needs to be regarded as a pilot, which will enable more accurate power calculations to be performed in the future. The primary outcome measure chosen is pain relief as is the norm for pain trials, yet it may be that other outcomes such as improved quality of life due to reduced side effects may be of greater importance. Many patients report that the "odd twinge" does not significantly impact on their lives, but tiredness, impaired cognitive function and ataxia from the high-dose medication has a significant impact on activities of daily living.

Most centers specialized in the treatment of patients with TN do not have a large enough patient base of the right type of patients including those willing to have their medication regimens changed.

A variety of strategies has been suggested to increase recruitment to trials of both participants and clinicians, and a recent systematic review suggests increased education of clinicians about the benefits of RCTs is needed [43]. Thus, the sites chosen have a special interest in TN with experienced clinicians, and it is hoped they will be able to recruit the appropriate patients. It takes time to recruit patients to clinical trials, and there are few data available on this topic. A recent study of an orthodontics multicenter RCT showed that it took on average 19 min to recruit a patient to the study and then a further $110 \mathrm{~min}$ per patient to fully recruit to the study and ensure all administrative data were available [44]. The variations between the centers were large in respect to the administrative data time as it often depended on the research support staff that was available. There was also considerable time involved in meetings of the principal investigators, and the authors suggest that these timings are potentially much higher in more complex studies.

To improve recruitment to this trial ethics approved data for patients were prepared and distributed to clinicians attending relevant conferences and for placement in a Trigeminal Neuralgia Support group newsletter in the UK. Recruitment of patients could be problematical for other reasons that are specific to this condition. TN causes very severe pain, and once stabilized on medications patients are often reluctant to change for fear their pain will return or become unmanageable. They may have used a range of other AEDs including the two permitted ones in the past and so be reluctant to go back to them. Currently the most effective AEDs cannot be used. Patients with classical TN who have neurovascular compression of the nerve (seen on MRI) may opt to have an operation rather than try further medications as the outcomes from surgery can be better [15]. The population most at risk is the elderly, and they are likely to have medical conditions that preclude their inclusion in the trial.

Different disease definitions as well as imprecise allocation of non-standardized diagnostic criteria make 
comparison of the different patient populations virtually impossible. Many trials do not refer to any particular diagnostic criteria at all but are content to describe their patient as having classical $\mathrm{TN}$ without further clarification or definition of that term. This study uses the international classification of headache disorders (ICHD-3beta) and an experienced data management committee to ensure a standardized patient selection and prevent non-classical TN patients entering the trial.

Incomplete or insufficiently defined outcome parameters, which were not clearly stated in many of the RCTs conducted until now, is another serious problem. Many trials failed to report dropouts or withdrawals, while those that did report these measures did not use intentionto-treat analysis to analyze their study results. This is particularly problematic since the dropout and withdrawal rates in most $\mathrm{TN}$ trials are very high. Information on demographics and clinical characteristics of the different treatment groups is often incomplete and lacks a clear comparison of baseline data with regard to gender, age, duration of disease and pain scores.

Allocation concealment and blinding are often problematic in randomized controlled trials on $\mathrm{TN}$. Often blinding methods are not reported in detail, so that it remains unknown who was blinded to what. All of these trials stated that they were randomized, but most did not describe the utilized method of randomization or whether the randomization method used was effective in regard to homogenizing the groups that were to be compared in the final analysis.

Selective reporting is generally considered a minor problem in these small and often confined studies. Some studies however did not clearly state what assessment tools they used for their analysis, while others did not report the adverse events or side effects of the study drug consistently. The possibility of patients going into spontaneous remission with treatment or without was not raised by most authors when interpreting their data.

Other potential sources of bias were often underreported. Many studies use the investigated drug as add-on to carbamazepine or other established treatment regimens [34,43,45-49]. Considering the pharmacologic profile of carbamazepine and other frequently used antiepileptic drugs with many drug interactions and hepatic metabolism, this may also pose serious consequences to the trial outcome and data interpretation of these trials that we are only starting to marginally comprehend. One of the largest RCTs comparing carbamazepine and oxcarbazepine in 46 patients has only been reported as a conference abstract, and the other study, possibly the same one in a German journal, included 48 patients [50]. The EERW design is new, and there will be few trials against which to compare the results, but the outcome measures being used are from the range of those suggested by Dworkin et al. [33]. The recently extended Brief Pain Inventory [35] has been psychometrically tested but has not yet been tested in terms of sensitivity to change; however, the first section has been used in extensive analgesic trials and so outcomes can be compared.

This protocol is being published prior to the study being completed in accordance with the recent literature [51] so that it will be possible to compare what was intended and what was actually done, and it has been registered on a trials register since February 2012, which may help to recruit patients. A comparison of registered and published primary outcome measures in RCTs shows selective outcome reporting is still prevalent [52], but this may also be due to an incorrect choice of outcome measures. In this study it is difficult to determine how large a change can be expected as there are currently so few data in the RCTs on TN, and unlike other pain trials, $100 \%$ pain relief can be achieved as opposed to the usual $50 \%$.

The results of the trial will be published and presented at conferences including those for patients.

\section{Trial status}

Ongoing

\section{Additional files}

Additional file 1: TGN expert summary Dec 2010.

Additional file 2: Ethics committees.

\section{Abbreviations}

AED: Antiepileptic drugs; AE: Adverse event; CBZ: Carbamazepine; Bid: Twice daily; CRO: Contract research organization; EERW: Enhancement enriched randomized withdrawal designs; ICHD: International classification of headache disorders; DB: Double blind; DMC: Data monitoring committee; MHRA: Medicines and healthcare products regulatory agency UK; RCT: Randomized controlled trial; Tid: Three times daily; TN: Trigeminal neuralgia; SAE: Serious adverse event.

\section{Competing interests}

JZ, DE and MO received payments for consultancy work on the data monitoring committee. KG, JP, GG, VM and ST are full-time employees of Convergence Pharmaceuticals, Ltd. The authors declare that they have no competing interests.

\section{Authors' contributions}

JZ participated in the design and coordination, was head of the data monitoring group and drafted the paper. DE and $\mathrm{MO}$ participated in the design of the data monitoring group, monitored the whole trial from the clinical standpoint and contributed to drafting the paper. KG and JP conceived the study, participated in the design and coordination, and drafting of the paper. GG, VM and ST were responsible for the chemistry and pharmaceutical development, preclinical studies and electrophysiological characterization of CNV1014802 and the drafting of the paper. All authors read and approved the final manuscript.

\section{Authors' informations}

$J Z$ is the current head of the largest UK multidisciplinary facial pain unit and a pain medicine specialist with a particular interest in trigeminal neuralgia, conducted both medical and surgical trials and Cochrane author 
of systematic reviews on trigeminal neuralgia. JP is Head of Clinical Operations at Convergence Phamaceuticals, Ltd. DE is the Head of the Interdisciplinary Orofacial Pain Unit of the University of Zurich, Switzerland. MO is the scientific head of the West-German Headache Center and consultant neurologist at the Department of Neurology at the University Hospital Essen, University of Duisburg-Essen. GG is Head of Chemistry and Preclinical Development at Convergence Pharmaceuticals, Ltd. VM is Head of Electrophysiology at Convergence Pharmaceuticals, Ltd. ST is Chief Scientific Officer at Convergence Pharmaceuticals, Ltd. KG is Medical Director at Convergence Pharmaceuticals, Ltd.

\section{Acknowledgments}

JZ undertook some of the work at UCL/UCLHT and received a proportion of funding from the Department of Health's NIHR Biomedical Research Centre funding scheme. Sandra Starck, Mattias Grossman, Joachim Kind, Emma Pinnick, Loryn Thorburn and Maria Carnemolla from Parexel Inc. and Lynn Purkins from Purkins Pharma Consulting, Ltd., are involved in the operational management of the trial. John Kirkpatrick from Insight Statistical Consulting, Ltd., provides statistical consultancy and analysis planning. The study is fully funded by Convergence Pharmaceuticals, Ltd.

\section{Author details}

${ }^{1}$ Facial pain unit, Division of Diagnostic, Surgical and Medical Sciences, Eastman Dental Hospital, UCLH NHS Foundation Trust/University College London, 256 Gray's Inn Road, WC1X 8LD, London, UK. ${ }^{2}$ Convergence Pharmaceuticals Ltd, Maia Building, Babraham Research Campus, CB22 3AT, Cambridge, UK. ${ }^{3}$ Interdiscipilinary Orofacial Pain Unit, Clinic of Masticatory Disorders, Removable Prosthodontics, Geriatric and Special Care Dentistry, Center of Dental Medicine, University of Zurich, Plattenstrasse 11, CH-8032, Zurich, Switzerland. ${ }^{4}$ Department of Neurology and German Headache Center, University of Duisburg-Essen, Hufelandstr. 55, 45147, Essen, Germany.

Received: 2 October 2013 Accepted: 13 November 2013 Published: 23 November 2013

\section{References}

1. Obermann M, Yoon MS, Dommes P, Kuznetsova J, Maschke M, Weimar C, Limmroth V, Diener HC, Katsarava Z: Prevalence of trigeminal autonomic symptoms in migraine: a population-based study. Cephalalgia 2007, 27(6):504-509.

2. Katusic S, Williams DB, Beard CM, Bergstralh EJ, Kurland LT: Epidemiology and clinical features of idiopathic trigeminal neuralgia and glossopharyngeal neuralgia: similarities and differences, Rochester, Minnesota, 1945-1984. Neuroepidemiology 1991, 10(5-6):276-281.

3. van Kleef M, van Genderen WE, Narouze S, Nurmikko TJ, van Zundert J, Geurts JW, Mekhail N: 1. Trigeminal neuralgia. Pain Pract 2009, 9(4):252-259.

4. Koopman JS, Dieleman JP, Huygen FJ, De Mos M, Martin CG, Sturkenboom MC: Incidence of facial pain in the general population. Pain 2009, 147(1-3):122-127.

5. IHS: IHS Classification ICHD-111 Beta version. 2013, 33(9):629-808.

6. Obermann M, Yoon MS, Ese D, Maschke M, Kaube H, Diener HC, Katsarava Z: Impaired trigeminal nociceptive processing in patients with trigeminal neuralgia. Neurology 2007, 69(9):835-841.

7. Prasad S, Galetta S: Trigeminal neuralgia: historical notes and current concepts. Neurologist 2009, 15(2):87-94.

8. Zakrzewska JM: Diagnosis and differential diagnosis of trigeminal neuralgia. Clin J Pain 2002, 18(1):14-21.

9. Wu CJ, Lian YJ, Zheng YK, Zhang HF, Chen Y, Xie NC, Wang LJ: Botulinum toxin type $\mathrm{A}$ for the treatment of trigeminal neuralgia: results from a randomized, double-blind, placebo-controlled trial. Cephalalgia 2012, 32(6):443-450

10. Rothman KJ, Monson RR: Survival in trigeminal neuralgia. J Chronic Dis 1973, 26(5):303-309.

11. Taylor JC, Brauer S, Espir MLE: Long-term treatment of trigeminal neuralgia. Postgrad Med J 1981, 57:16-18.

12. Beydoun A, Schmidt D, D'Souza D: Oxcarbazepine versus carbamazepine in trigeminal neuralgia : a meta-anlaysis of three double blind comparative trials. [abstract]. Neurol 2002, 58:p02.083.

13. Anonymous: The international classification of headache disorders: 2 nd edition. Cephalalgia 2004, 24(Suppl 1):9-160.
14. Anon: The international classification of headache disorders, 3rd edition (beta version). Cephalalgia 2013, 33(9):629-808.

15. Cruccu G, Gronseth G, Alksne J, Argoff C, Brainin M, Burchiel K, Nurmikko T, Zakrzewska JM: AAN-EFNS guidelines on trigeminal neuralgia management. Eur J Neurol 2008, 15(10):1013-1028.

16. Obermann M, Yoon MS, Sensen K, Maschke M, Diener HC, Katsarava Z Efficacy of pregabalin in the treatment of trigeminal neuralgia. Cephalalgia 2008, 28(2):174-181.

17. Wiffen PJ, Derry S, Moore RA, McQuay HJ: Carbamazepine for acute and chronic pain in adults. Cochrane Database Syst Rev 2011, 1:CD005451.

18. Meador KJ: Cognitive and memory effects of the new antiepileptic drugs. Epilepsy Res 2006, 68(1):63-67.

19. Meador KJ, Gevins A, Loring DW, McEvoy LK, Ray PG, Smith ME, Motamedi GK, Evans BM, Baum C: Neuropsychological and neurophysiologic effects of carbamazepine and levetiracetam. Neurology 2007, 69(22):2076-2084.

20. Motamedi GK, Meador KJ: Antiepileptic drugs and memory. Epilepsy Behav 2004, 5(4):435-439

21. Moriarty $\mathrm{O}, \mathrm{McGuire} B \mathrm{BE}$, Finn DP: The effect of pain on cognitive function: a review of clinical and preclinical research. Prog Neurobiol 2011, 93(3):385-404

22. Fischer TZ, Waxman SG: Familial pain syndromes from mutations of the NaV1.7 sodium channel. Ann N Y Acad Sci 2010, 1184:196-207.

23. Faber CG, Hoeijmakers JG, Ahn HS, Cheng X, Han C, Choi JS, Estacion M, Lauria G, Vanhoutte EK, Gerrits MM, Dib-Haji S, Drenth JP, Waxman SG, Merkies IS: Gain of function Nanu1.7 mutations in idiopathic small fiber neuropathy. Ann Neurol 2012, 71(1):26-39.

24. Pietrobon D: Insights into migraine mechanisms and CaV2.1 calcium channel function from mouse models of familial hemiplegic migraine. J Physiol 2010, 588(Pt 11):1871-1878.

25. Moore A, Derry S, Eccleston C, Kalso E: Expect analgesic failure; pursue analgesic success. BMJ 2013, 346:f2690

26. Chan AW, Tetzlaff JM, Gotzsche PC, Altman DG, Mann H, Berlin JA, Dickersin K, Hrobjartsson A, Schulz KF, Parulekar WR, Krleza-Jeric K, Laupacis A, Moher D: SPIRIT 2013 explanation and elaboration: guidance for protocols of clinical trials. BMJ 2013, 346:e7586

27. Jorns TP, Zakrzewska JM: Evidence-based approach to the medical management of trigeminal neuralgia. Br J Neurosurg 2007, 21(3):253-261.

28. Wiffen PJ, Derry S, Moore RA: Lamotrigine for acute and chronic pain. Cochrane Database Syst Rev 2011, 2(2):CD006044.

29. Yang M, Zhou M, He L, Chen N, Zakrzewska JM: Non-antiepileptic drugs for trigeminal neuralgia. Cochrane Database Syst Rev 2011, 1:CD004029.

30. McQuay HJ, Derry S, Moore RA, Poulain P, Legout V: Enriched enrolment with randomised withdrawal (EERW): time for a new look at clinical trial design in chronic pain. Pain 2008, 135(3):217-220.

31. Quessy SN: Two-stage enriched enrolment pain trials: a brief review of designs and opportunities for broader application. Pain 2010, 148(1):8-13.

32. Jorns TP, Johnston A, Zakrzewska JM: Pilot study to evaluate the efficacy and tolerability of leviteracetam (keppra) in the treatment of patients with trigemional neuralgia. Eur I Neurol 2009, 16:740-744.

33. Dworkin RH, Turk DC, Farrar JT, Haythornthwaite JA, Jensen MP, Katz NP, Kerns RD, Stucki G, Allen RR, Bellamy N, Carr DB, Chandler J, Cowan P, Dionne R, Galer BS, Hertz S, Jadad AR, Kramer LD, Manning DC, Martin S, McCormick CG, McDermott MP, McGrath P, Quessy S, Rappaport BA, Robbins W, Robinson JP, Rothman M, Royal MA, Simon L, Stauffer JW, Stein W, Tollett J, Wernicke J, Witter J: Core outcome measures for chronic pain clinical trials: IMMPACT recommendations. Pain 2005, 113(1-2):9-19.

34. Zakrzewska JM, Chaudhry Z, Nurmikko TJ, Patton DW, Mullens EL: Lamotrigine (lamictal) in refractory trigeminal neuralgia: results from a double-blind placebo controlled crossover trial. Pain 1997, 73(2):223-230.

35. Lee JY, Chen HI, Urban C, Hojat A, Church E, Xie SX, Farrar JT: Development of and psychometric testing for the brief pain inventory-facial in patients with facial pain syndromes. J Neurosurg 2010, 113(3):516-523.

36. Baker GA, Frances P, Middleton E: Initial development, reliability, and validity of a patient-based adverse event scale. Epilepsia 1994, 35(suppl 7):80.

37. Gilliam FG, Fessler AJ, Baker G, Vahle V, Carter J, Attarian H: Systematic screening allows reduction of adverse antiepileptic drug effects: a randomized trial. Neurology 2004, 62(1):23-27.

38. Hays RD, Sherbourne CD, Mazel RM: User's manual for medical outcomes study (MOS) Core Measures of Health-Related Quality of Life, Rand Corporation MR-162-RC. Santa Monica; 1995.

39. Stewart AL, Ware JE, Sherbourne CD: Psychological distress/well-being and cognitive functioning measures. In Measuring Functioning and Well-being: The 
Medical Outcomes Study Approach. Edited by Stewart AL, Ware JE. Durham NC: Duke University; 1992:102-142.

40. Perucca P, Carter J, Vahle V, Gilliam FG: Adverse antiepileptic drug effects: toward a clinically and neurobiologically relevant taxonomy. Neurology 2009, 72(14):1223-1229.

41. Moher D, Jones A, Lepage L: Use of the CONSORT statement and quality of reports of randomized trials: a comparative before-and-after evaluation. JAMA 2001, 285(15):1992-1995.

42. Gilron I, Booher SL, Rowan JS, Max MB: Topiramate in trigeminal neuralgia: a randomized, placebo-controlled multiple crossover pilot study. Clin Neuropharmacol 2001, 24(2):109-112.

43. Fletcher B, Gheorghe A, Moore D, Wilson S, Damery S: Improving the recruitment activity of clinicians in randomised controlled trials: a systematic review. BMJ Open 2012, 2(1):e000496.

44. Ellis PE, Bradley RL, Sandy JR, Deacon SA, Griffiths HS, Atack NE, Moore MB, House KA, Wenger NA, Worth V, Ireland AJ: Do I have enough time? The impact of recruiting patients to a randomised controlled trial at recruiting centres. Br Dent J 2012, 213(9):467-470.

45. Lechin F, van der Dijs B, Lechin ME, Amat J, Lechin AE, Cabrera A, Gomez F, Acosta E, Arocha L, Villa S, Jienez V: Pimozide therapy for trigeminal neuralgia. Arch Neurol 1989, 46:960-963.

46. Lindstrom $P$, Lindblom $V$ : The analgesic effect of tocainide in trigeminal neuralgia. Pain 1987, 28:45-50.

47. Vilming ST, Lyberg $T$, Latase X: Tizanidine in the management of trigeminal neuralgia. Cephalalgia 1986, 6:181-182.

48. Shaikh S, Yaacob HB, Abd Rahman RB: Lamotrigine for trigeminal neuralgia: efficacy and safety in comparison with carbamazepine. J Chin Med Assoc 2011, 74(6):243-249.

49. Lemos L, Flores S, Oliveira P, Almeida A: Gabapentin supplemented with ropivacain block of trigger points improves pain control and quality of life in trigeminal neuralgia patients when compared with gabapentin alone. Clin J Pain 2008, 24(1):64-75.

50. Liebel JT, Menger N, Langohr H: Oxcarbazepine in der Behandlung der Trigeminusneuralgie. Nervenheilkd 2001, 20:461-465.

51. Godlee F: Publishing study protocols: making them visible will improve registration, reporting and recruitment. BMC News and Views 2001, 2:4.

52. Mathieu S, Boutron I, Moher D, Altman DG, Ravaud P: Comparison of registered and published primary outcomes in randomized controlled trials. JAMA 2009, 302(9):977-984.

doi:10.1186/1745-6215-14-402

Cite this article as: Zakrzewska et al:: Novel design for a phase lla placebo-controlled, double-blind randomized withdrawal study to evaluate the safety and efficacy of CNV1014802 in patients with trigeminal neuralgia. Trials 2013 14:402.

\section{Submit your next manuscript to BioMed Central and take full advantage of:}

- Convenient online submission

- Thorough peer review

- No space constraints or color figure charges

- Immediate publication on acceptance

- Inclusion in PubMed, CAS, Scopus and Google Scholar

- Research which is freely available for redistribution 